\title{
A High-Granularity Timing Detector for the Phase-II upgrade of the ATLAS Detector System
}

\author{
Christina Agapopoulou on behalf of the ATLAS LAr-HGTD group \\ Universite Paris Sud \\ Laboratoire de l'Accelerateur Lineaire \\ Orsay, France \\ Email: agapopoulou@lal.in2p3.fr
}

\begin{abstract}
The expected increase of the particle flux at the high luminosity phase of the LHC with instantaneous luminosities up to $L=7.5 \times 10^{34} \mathrm{~cm}^{-2} \mathrm{~s}^{-1}$ will have a severe impact on pile-up. The pile-up is expected to increase on average to 200 interactions per bunch crossing. The reconstruction performance for especially jets and transverse missing energy will be severely degraded in the end-cap and forward region of the ATLAS detector. A High Granularity Timing Detector (HGTD) is proposed in front of the liquid Argon end-cap calorimeters of ATLAS for pile-up mitigation in the offline reconstruction. An additional use of the detector as a luminometer is proposed. This device covers the pseudo-rapidity range of 2.4 to about 4. Four layers of Silicon sensors are foreseen to provide precision timing information with a time resolution of the order of 30 pico-seconds per minimum ionizing particle in order to assign the energy deposits in the calorimeter to different proton-proton collision vertices. Each readout sensor has a transverse size of only a few $\mathbf{m m}$, leading to a highly granular detector with several million readout channels. The expected improvements in performance are relevant for physics processes, i.e, vector-boson fusion and vector-boson scattering processes, and for physics signatures with large missing transverse energy. The chosen silicon sensor technology is Low Gain Avalanche Detectors (LGAD). In this document, starting from the physics motivations of the High Granularity Timing Detector, the proposed detector layout and Front End readout, laboratory and beam test characterization of sensors and the results of radiation tests will be discussed.
\end{abstract}

\section{Motivation}

In 2026, LHC will enter its High Luminosity phase, where the instantaneous luminosity will increase from $2-3 \times 10^{34}$ $\mathrm{cm}^{-2} \mathrm{~s}^{-1}$ to $7.5 \times 10^{34} \mathrm{~cm}^{-2} \mathrm{~s}^{-1}$ [1]. One of the main challenges of HL-LHC will be the management of pile-up interactions, which are all the interactions happening around the interaction of interest. With the increased luminosity, an average of 200 pile-up interactions per event are expected, while the beam average spread is expected to be $50 \mathrm{~mm}$ along the beam axis. Fig. 1 shows the expected resolution of the $z_{0}$ impact parameter used for track-to-vertex association as a function of the track pseudorapidity for tracks with different $\mathrm{p}_{\mathrm{T}}$. The impact parameter resolution grows rapidly as a function of the track $|\eta|$ and, in the forward region, beyond $|\eta|=2.5$, it reaches very large values of several millimeters. The resolution also worsens at low $\mathrm{p}_{\mathrm{T}}$ due to multiple scattering effect. In HL-LHC conditions, the local average vertex density is expected to be approximately 1.6 vertices $/ \mathrm{mm}$ reaching up to 3 vertices $/ \mathrm{mm}$ in certain occasions.

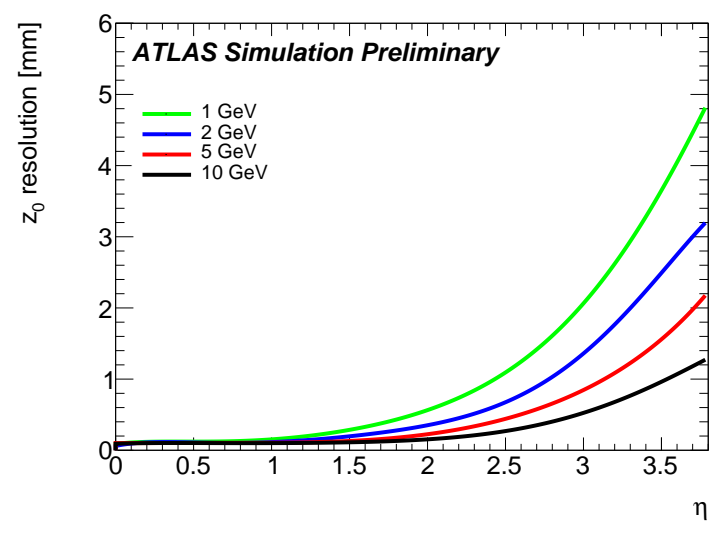

Fig. 1: Longitudinal track impact parameter $\mathrm{z}_{0}$ resolution as a function of $\eta$ for different $\mathrm{p}_{\mathrm{T}}$ values

Under such a high density of interactions, distinguishing the individual vertices, finding the primary vertex and matching the tracks to the vertices only using the spacial resolution of the Inner Tracker (ITk) of ATLAS becomes a challenge. As a consequence the accuracy of the reconstruction of all physics objects is expected to be degraded. This effect is especially pronounced in the forward region, where the particle density is the largest. The forward region contains physics signatures of great interest, such as Vector Boson Fusion (VBF) Higgs production, where forward jets provide the key handle for the processs trigger.

The HGTD gives a solution to this problem, since the additional time information it provides can be used to distinguish "merged" pile-up vertices that are in a distance below the spatial sensitivity of the Inner detector. In the HL-LHC, the collisions are expected to follow a Gaussian distribution in time with $\sigma=180 \mathrm{ps}$. Given the current estimate for the time resolution of the HGTD is $30 \mathrm{ps}$, the detector can greatly help in the mitigation of pile-up. In addition, the HGTD can be used as a precise luminometer. Due to its high granularity, it is expected to provide good linearity between the number of hits and the number of interactions, while adjustable time windows can be used to study afterglow effects. 


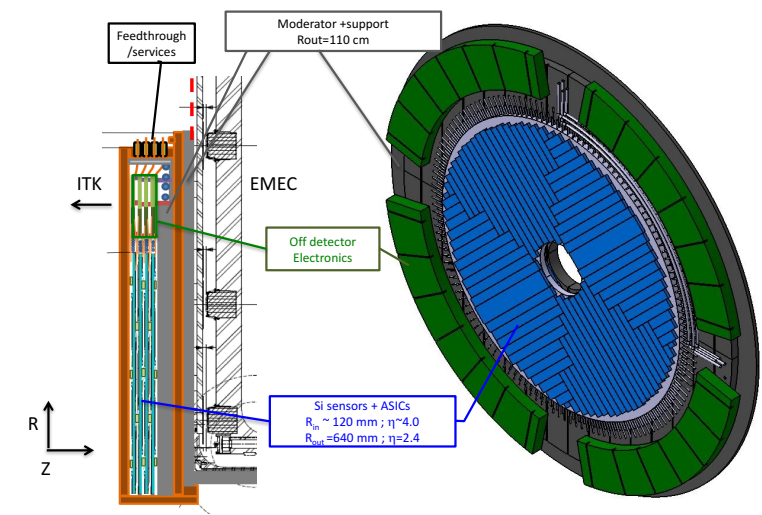

Fig. 2: Illustration of the HGTD detector. On the left, the detector installed between the endcap calorimeter cryostat and the ITk. On the right, the different components of the HGTD. The central blue parts correspond to the active area. The green blocks correspond to the off-detector electronics. The grey cylinder behind is the moderator needed to shield the backscattered neutrons from the endcap calorimeter.

\section{Detector OVERVIEW}

The main constraints for the HGTD are the limited available space in ATLAS and the harsh radiation environment of HLLHC. The total envelope of the HGTD, including off-detector electronics and support structures, will ocuppy a space between $\mathrm{R}=110-1100 \mathrm{~mm}$ in radius, while a volume that ranges from 2.4 to 4 in $|\eta|$ or equivalently $120-640 \mathrm{~mm}$ in radius is currently considered free for the active area of the detector. To satisfy the space limitations in ATLAS, the HGTD is proposed to be a very thin, disk-shaped planar detector, occupying the forward region on both sides at $|z|=(3420,3545) \mathrm{mm}$, as illustrated in Fig. 2. HGTD will be placed between the ITK and $50 \mathrm{~mm}$ of moderator (used to protect the ITk and HGTD from radiated neutrons coming from the calorimeters). The lack of space is a limiting factor also for the number of layers of the detector and the design of the services, such as electronic readout and cooling. The current design includes 4 layers to improve the time resolution, while the possibility to have 2 or 3 layers is being explored for the best compromise between performance and cost.

In order to be able to reconstruct individually most of the particles hitting the detector, a low occupancy is also necessary. An upper limit of $10 \%$ for the occupancy can be achieved by making the detector very granular. The current choice is to have $1.3 \times 1.3 \mathrm{~mm}^{2}$ sensors, while a $1 \times 1 \mathrm{~mm}^{2}$ size option is still under study.

Following the constraints and requirements previously described, silicon has been chosen as the sensor material for the detector, since it can provide sufficient time resolution, high granularity and compactness. The signal from the sensors will be readout by $2 \times 2 \mathrm{~cm}^{2} 225$-channel Application-specific integrated circuits (ASICs). The connection will be established by directly bump-bonding the sensors to the ASICs. The ASIC-sensor units will be organized in $2 \times 4 \mathrm{~cm}^{2}$ modules containing 2 ASICs each. The modules will then be placed on top of kapton flexes that will transfer the data from the ASICs to the off-detector electronics. The modules will be placed on both sides of the cooling plate with a small overlap of about $2 \mathrm{~mm}$, in order to minimize dead areas.

Due to the high particle rate in the forward region, it is also crucial for the HGTD to be radiation-hard to be able to function without the need for repair for a large period of time. With radiation levels reaching up to $9 \times 10^{15} \mathrm{n}_{\mathrm{eq}} / \mathrm{cm}^{2}$ and a Total Ionizing Dose (TID) of 9 MGy after 4000 $\mathrm{fb}^{-1}$ (integrated luminostiy at the end of HL-LHC) including safety factors, a replacement of the inner-radius $(\mathrm{R}<300$ $\mathrm{mm}$ ) modules is planned at the half-time of the HL-LHC. Consequently the HGTD will need to outstand radiation level that are half of the values mentionned above .

\section{SENSORS}

\section{A. Sensor Technology}

The best performing detector technology is the Low Gain Avalanche Detector (LGAD) [2], [3] which is a n-p silicon detector with a highly doped p-layer below the n-p junction. When a charged particle crosses the detector, it creates pairs of electrons and holes, which drift in opposite directions due to an externally applied voltage (bias voltage). When the electrons reach the doped layer, they produce more electrons and holes, inducing an avalanche which creates the amplified detected signal. The amplification gain of the LGAD sensors designed for the HGTD is around 20. The time resolution of the detector needs to be as good as possible, to allow for precise timing information. For the HGTD, the main contributions to the time resolution are given by the following equation:

$$
\sigma_{\text {det }}^{2}=\sigma_{\text {Landau }}^{2}+\sigma_{\text {electronics }}^{2}
$$

- the Landau fluctuations of the sensor, that are due to the non-uniformity of the particle energy deposit. Simulation results show that this contribution can be limited by chosing a thin sensor with medium doping. A sensor of about $50 \mu \mathrm{m}$ corresponds to a Landau fluctuation of about $25 \mathrm{ps}$, which makes the goal of a 30 ps time resolution achievable.

- the electronics contribution, which can be traced back to three main factors:

(1) The jitter, that is the noise contribution to the signal, is given by the expression:

$$
\sigma_{\text {jitter }}=\frac{N}{d V / d t}
$$

where $N$ is the noise and $d V / d t$ the slope of the signal. For a given signal amplitude, thin sensors have a faster rising time and therefore are optimal for the jitter suppression. Additionally, the jitter is minimized for small detector capacitance, which is achieved by making smallarea sensors $(C \propto A / d$, where $A=$ area and $d=$ thickness of the sensor). On the other hand, the detector capacitance contribution to the noise is a limiting factor to the thinness of the sensor. 
(2) The Time Walk which is basically the error due to the fact that, when measuring time with a constant threshold discriminator, high-amplitude signals cross the discriminator constant threshold faster than smaller ones. This effect produces a dependence of the digital signal on the amplitude of the analog one, but can be corrected by using a Time-over-Treshold (TOT) discriminator, which measures the width of the pulse that is proportional to the analog signal amplitude. After correction, the time walk is expected to give a negligible contribution, smaller than 10 ps RMS peak-to-peak.

(3) Time to digital converter (TDC) binning. The TDC uses delaying cells to compare between a fast STOP signal and a slow START signal. The fast STOP signal enters the delay line and the amount of cells needed to delay it so that it becomes in-time with the START signal corresponds to the time measurement. This method introduces a resolution equal to the cell delay value, which, for the HGTD, is 20 ps. The final contribution of the TDC, $\sigma_{\mathrm{TDC}}=20 \mathrm{ps} / \sqrt{12}$, is negligible.

\section{B. Sensor Production and Testing}

The LGAD technology was proposed and developed by the Centro Nacional de Microelectronica (CNM) in Barcelona. LGAD sensor production started in 2014 by CNM [4], Hamamatsu Photonics (HPK) and Fondazione Bruno Kessler (FBK). Sensors were produced in single pads and arrays with various sizes, thicknesses and doping concentrations, in order to find the optimal design.

The produced sensors were tested by various lab stations. $\mathrm{I}-\mathrm{V}$ and $\mathrm{C}-\mathrm{V}$ setups were used for the electric characterization of the devices, while beta-source $\left({ }^{90} \mathrm{Sr}\right)$, laser and testbeam measurements were performed to estimate the LGAD dynamic properties, such as gain and time resolution [5], [6]. The beam test campaigns started in August 2016 and are still ongoing, with the last one having been completed in September 2017. All of them were performed at CERN, at the lines H6A and H6B of the SPS, using $120 \mathrm{GeV}$ pions.

\section{Results}

Un-irradiated Sensors: Different un-irradiated sensors showed fairly homogenous electrical behavior. The gain, which is defined as the charge collected by the LGAD, divided by the charge collected by a no-gain PIN diode without multiplication layer, was found to increase as a function of the bias voltage and the doping concentration, due to the enhancement of the amplification field. Measurements at different temperatures also show that the gain is inversely proportional to the temperature, due to higher impact ionization [7]. The time resolution of a LGAD was extracted using either a second LGAD or a cherenkov quartz counter attached to a SiPM as a time reference. Results from the Autumn 2016 beam test campaign for various pads and arrays are shown in Fig. 3, where the time resolution can be viewed as a function of the gain. For single pads, a fairly uniform behavior is observed: the time resolution reaches a plateau for gain higher than 20 ,

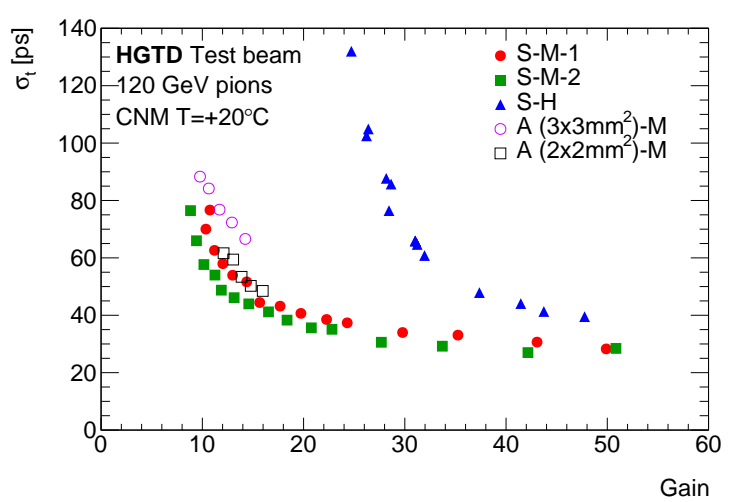

Fig. 3: Time resolution as a function of the gain for single pad sensors (filled points) and arrays (empty points). For single pads, the time resolution reaches a plateau for gain higher than 20, and the best performance is achieved for medium doping (S-M-1 and 2). Measurements with S-M-2 have been performed using a different preamplifier than S-M-1, resulting in minor improvement. Arrays A2M and A3M cannot operate at gain $>15$ due to earlier breakdown. The time resolution at a given gain is worse for arrays than for single pads because of the larger detector capacitance. Statistical uncertainties are negligible and smaller than the marker size.

and the best performance is achieved for medium doping. The arrays, on the other hand, experience breakdown at much lower bias voltage, and therefore cannot operate at a gain of more than 15. This limitation decreases their performance and is due to the lack of a guard ring in the interpad regions. In addition, due to the larger detector capacitance, they exhibit worse jitter at a given gain than the single pad sensors.

After Irradiation: To estimate the performance of the HGTD in the harsh radiation environment of the HL-LHC, the sensors were irradiated with neutrons at various fluences up to $6 \times 10^{15}$ $\mathrm{n}_{\mathrm{eq}} / \mathrm{cm}^{2}$ at the JSI research reactor in Ljubliana. Measurements on irradiated sensors [6] showed a reduction of gain, due to the removal of active dopants from the amplification layer. In order to maintain some of the original gain, an operation at higher $\mathrm{V}_{\text {bias }}$ is necessary. In addition, the observed leakage current increases, which calls for an operation at lower temperature, around $\mathrm{T}=-20-30{ }^{\circ} \mathrm{C}$. Another interesting effect of the irradiation that was observed was the decrease of the rise time of the signal, which is attributed to active defects in the bulk of the sensor that cause multiplication outside the amplification layer. As shown in Fig. 4, the time resolution increases as a function of the fluence due to the loss of gain, but does not exceed the 60 ps limit. Therefore, it is expected that LGAD sensors will meet the timing requirements up to the half-life of HL-LHC.

\section{FRONT-END EleCtRonics}

The LGAD signal will be read out and digitized by a 225 channels, $2 \times 2 \mathrm{~cm}^{2}$ ASIC on which the sensor will be bumpbonded. The design of the ASIC should be optimized to min- 


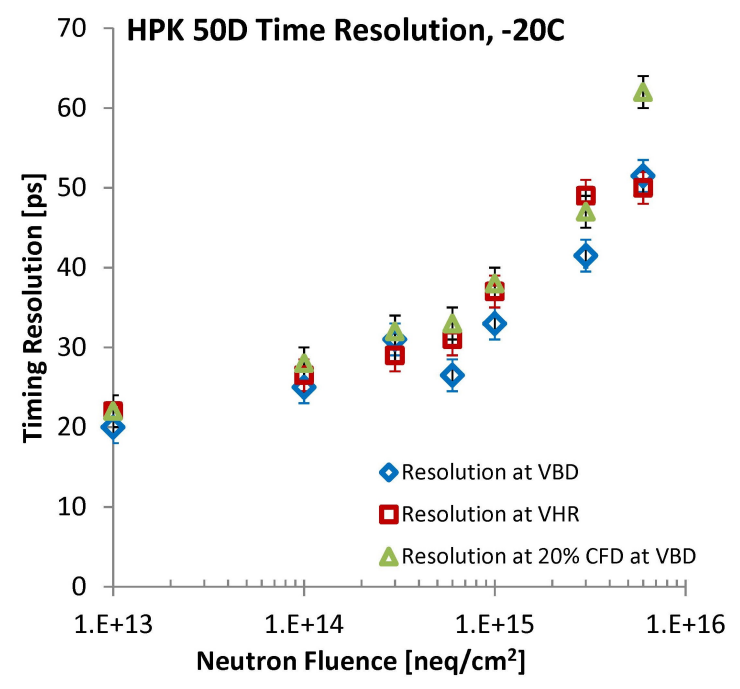

Fig. 4: Fluence dependence of the time resolution. Different markers correspond to different bias voltages and constantfraction-discriminator (CFD) methods used (offline) to correct for the time walk effect.

imize the electronic noise contribution to the time resolution while withstanding the radiation environment of the HL-LHC. In addition, the power dissipation should be minimized, to maintain the cooling budget. A power consumption of less than $200 \mathrm{~mW} / \mathrm{cm}^{2}$ is currently considered as a reasonable goal. Each channel of the ASIC, as shown in Fig. 5, will contain:

- a preamplifier based on the $130 \mathrm{~nm}$ TSMC CMOS technology, with a variable bandwidth of 3-5 GHz, followed by

- a discriminator that provides a Time-Over-Threshold measurement (TOT-pulse width above threshold). This measurement will be used offline to correct for the Time Walk effect

- two Time-to-Digital Converters (TDC) for the digitization of Time-of-Arrival and Time-Over-Threshold measurements

- a Local FIFO memory, storing the information until the trigger signal.

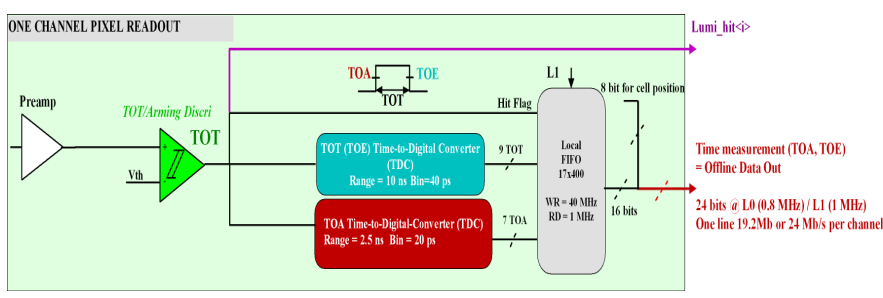

Fig. 5: Schematic of a single readout pad

\section{A. Prototype Measurements}

A first prototype of this dedicated ASIC, named ALTIROC0 [8] was designed by Omega and the first 7 boards were received for testing in March 2017. This prototype is an 8channel chip which integrates only the preamplifier and the discriminator in each channel. The digital part of the front end electronics has not been included, in order to test the analog characteristics of the chip.

First tests of the prototype were performed at the Omega testbench, in June 2017. The ALTIROC0 was wire-bonded on a testboard without any sensors and a picosecond generator was providing a test pulse with a typical rise-time of 100 ps. The power consumptions of the preamplifier and of the discriminator were measured respectively $420 \mu \mathrm{W}$ and 375 $\mu \mathrm{W}$. In order to perform measurements in more realistic conditions, a capacitance was soldered on the board, replacing the detector capacitance. The value of this capacitance varied during the testing, to estimate the chip's performance for different sensor designs. This setup allowed the study of the electronic's jitter as a function of the injected charge, as shown in Fig. 6, where a $2 \mathrm{pF}$ capacitance was used (at the time of the testing, the most probable size of the pads was $1 \times 1 \mathrm{~mm}^{2}$, corresponding to this capacitance value). As expected, the jitter is decreasing for higher charge, due to the larger induced signals. For $10 \mathrm{fC}$, a value that corresponds to approximately $1 \mathrm{MIP}$, the jitter was found to be $27 \mathrm{ps}$, while after $80 \mathrm{fC}$, it reaches a 7 ps plateau due to the resolution of the generator. The parasitic capacitance due to the board was estimated to be $1.3 \mathrm{pF}$, which should be added to the soldered capacitance.

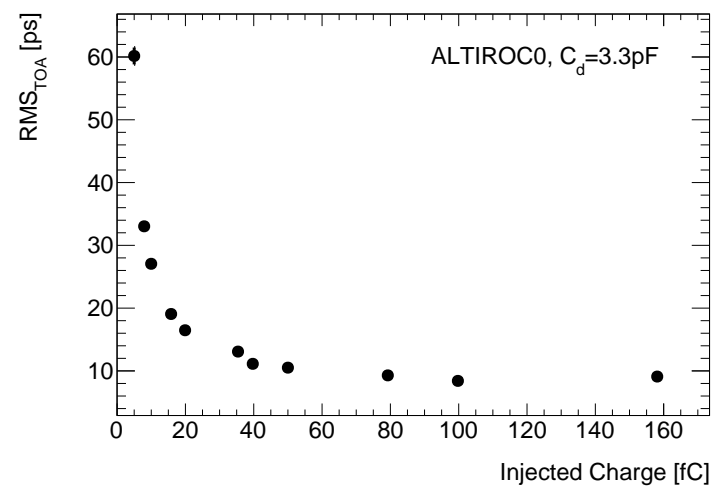

Fig. 6: Jitter RMS as a function of the injected charge with $2 \mathrm{pF}$ soldered capacitance. An estimated $1.3 \mathrm{pF}$ parasitic capacitance should be added.

In September 2017, the prototype was also tested at the CERN testbeam lines H6A and H6B of the SPS, using $120 \mathrm{GeV}$ pions. The tested board included the ALTIROC0 prototype with a $2 \times 2$ unirradiated bump-bonded sensor array of $1 \times 1 \mathrm{~mm}^{2}$ sensors. Results from this campaign are very preliminary, but indicate that the preamplifier is slower than expected. In addition, the detector capacitance was found to be $4 \mathrm{pF}$, higher than the expected $2 \mathrm{pF}$ value $(3.3 \mathrm{pF}$ including the board parasitics). The reason for this difference is still under investigation, but the most probable reason is that the active sensor is actually thinner than $50 \mu \mathrm{m}$, while interpad capacitance might also be contributing. Fig. 7 shows 
the time resolution as a function of the pole capacitance of the preamplifier that controls its bandwidth. The best value of $47 \mathrm{ps}$ is achieved for the fastest setting of the preamplifier. An improved result should therefore be possible with a faster preamplifier. The next iteration of the ALTIROC will be integrating an optimized preamplifier design, an improved layout to minimize parasitic capacitance and the digital parts of the read-out (TDC and FIFO memory).

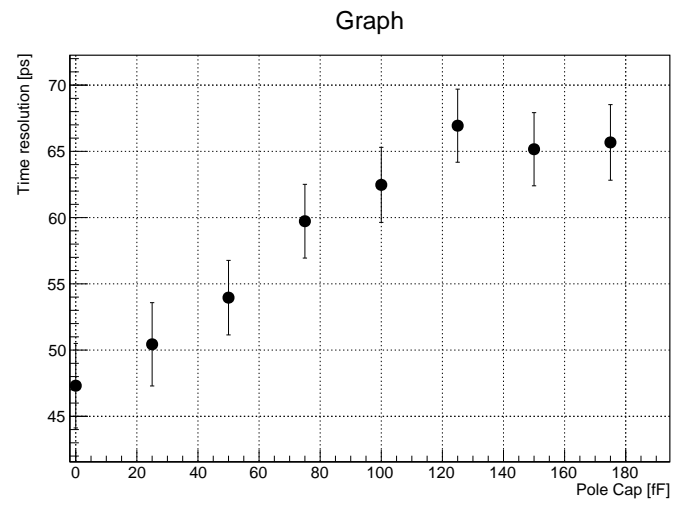

Fig. 7: Time resolution as a function of the pole capacitance of the preamplifier. The sensor bias voltage used was $V_{\text {bias }}=130$ $\mathrm{V}$. The best value of $47 \mathrm{ps}$ is found for the fastest setting of the preamplifier $\left(\mathrm{C}_{p}=0 \mathrm{fF}\right)$, indicating that a faster preamplifier might be optimal.

\section{CONCLUSION}

The HGTD is a detector proposed for the High-Luminosity Phase of the ATLAS experiment. The high granularity and precise time measurement will help mitigate the pile-up effects and improve the current reconstruction techniques by adding a new orthogonal observable, the time. Its requirements to be radiation hard, compact and granular are satisfied by $\mathrm{Si}$ detectors, while the high quality time measurement can be provided by the LGAD technology. Sensor tests in lab probes and beam test campaigns have shown that $\mathrm{Si}$ sensors with the LGAD technology can achieve a time resolution less than 30 ps before irradiation and less than 60 ps after irradiation, therefore satisfying the timing requirements. A first prototype of the front-end electronics ASIC, named ALTIROC0 has been fabricated. It includes 8 channels of only the analog parts of the design, the preamplifier and the discriminator. Preliminary tests attribute a 27 ps jitter when using a test pulse in testbench measurements, while this performance is degraded to $47 \mathrm{ps}$ at testbeam measurements with LGAD sensors bump-bonded to the ASIC. The results seem to indicate that the detector capacitance is larger than expected and that a faster preamplifier is needed. The next iteration of the ASIC, ALTIROC1, will integrate this improvement along with an optimized board layout for minimizing parasitic capacitance and the digital parts of the front-end electronics.

\section{ACKNOWLEDGMENT}

This research is the collective work of the Liquid Argon HGTD group. I would like to thank the LAr-HGTD group for their immense efforts in realising this project.

\section{REFERENCES}

[1] ATLAS Phase-II Upgrade Scoping Document, CERN-LHCC-2015-020. LHCC-G-166, CERN, 2015, https://cds.cern.ch/record/2055248

[2] G. Pellegrini et al., Technology developments and first measurements of Low Gain Avalanche Detectors (LGAD) for high energy physics applications, Nucl.Instrum.Meth.A765(2014)12

[3] H.-W. Sadrozinski, A. Seiden and N. Cartiglia, 4-Dimensional Tracking with Ultra-Fast Silicon Detectors, arXiv: 1704.08666

[4] M. Carulla et al., First 50 m thick LGAD fabrication at CNM, 28th RD50 Workshop, Torino, Italy, June 7th 2016, 2016, https://agenda.infn.it/getFile.py/access?contribId=20\&sessionId=8\& resId $=0$ \&materialId $=$ slides \& confId $=11109$

[5] N. Cartiglia et al., Beam test results of a 16 ps timing system based on ultra-fast silicon detectors, Nucl. Instrum. Meth. A850 (2017) 83, arXiv: 1608.08681[physics.ins-det].

[6] J. Lange et al., Gain and time resolution of $45 \mathrm{~m}$ thin Low Gain Avalanche Detectors before and after irradiation up to a fluence of $10^{15} n_{e q} / \mathrm{cm}^{2}$ , JINST 12 (2017) P05003, arXiv:1703.09004[physics.ins-det]

[7] D.J Massey et al, Temperature Dependence of Impact Ionization in Submicrometer Silicon Devices, IEEE Transactions on Electron Devices, vol. 53, no. 9, September 2006.

[8] C. de la Taille, ALTIROCO, a 20 Pico-Second Time Resolution ASIC for the ATLAS High Granularity Timing Detector (HGTD), 2017 Topical Workshop on Electronics for Particle Physics (TWEPP 2017) https://indico.cern.ch/event/608587/contributions/2614083/ 